\title{
Research to an alternative explaination of redshift
}

\author{
Luciano Rota
}

Sportmark 1041355 KH Almere Netherlands

E-mail: Imrrota@live.nl

Copyright $\odot 2014$ Luciano Rota. This is an open access article distributed under the Creative Commons Attribution License, which permits unrestricted use, distribution, and reproduction in any medium, provided the original work is properly cited.

\begin{abstract}
Is the "Big-Bang" really existed? The present research on theoretical base, through a correct and accurate analysis of the Doppler Effect, attempts to state that there is another concrete and sustainable way to understand the phenomenon of redshift, which could eradicate the hypothesis of an expanding universe.
\end{abstract}

Keywords: Redshift; Doppler-Shift; Magnetic Fields; Gravity; Slow Light.

\section{Introduction}

Around the twenties of the past century, astronomy made a discovery that led to eradication of a conviction till than considered obvious. From Galilean times, we can say that it was tacitly supposed that the universe, in its entirety, was static.

A group of astronomers, who had already at the beginning of 1900 started an analysis of the spectrum of the galaxies around ours, found themselves face to face with a disturbing phenomenon. The result of this research was the following: since the composition of light emanating from the celestial bodies very far from us presented the same characteristics as the stars closer to us, all the colors of the spectrum, at equal measure, shifted to red.

This phenomenon, known as redshift, considering the constancy of the speed of light, led to one only interpretation: the bodies examined move away from us in constant motion at a speed - and this is the surprising part of the discovery that is directly proportional to the distance at which they are. In other words: the further away a celestial body is, the faster it moves away from us. The interpretative key to this phenomenon is the Doppler Effect: when a source of waves moves away from its observer, increases the wavelength. It means that the distance between two wave-tops become bigger than that originally emitted.

The relation that in synthesis can be defined as "the farther the distance the faster the speed of moving away" was made public for the first time in 1929 by the astronomer Edwin Hubble.

Speculating on this fact, the hypothesis was made that the universe originated from an explosion of a very high concentration of matter, after which innumerable parts, disintegrating, would, in subsequent evolution, have given origin to the galaxies and therefore to the stars and the planets.

The "Big-Bang" theory is really not devoid of interrogatives. The one most shrouded in mystery is the following: for which reason, principle and energy source, the "splinters" of this gigantic explosion can increase their speed in relation to the starting point in the measure that they move away from it? Our real experience would hint the opposite: what we see daily is that every object that finds its kinetic thrust exhausts this energy with the distance, in a relation that is exactly the opposite of the one hypothesized by the "Big-Bang" theory.

There could be a different explanation for the redshift?

\section{Method}

We start analyzing the Doppler Effect in its classical concept and its consequences with regard to this matter:

1) Treating of classical waves, we have to consider that an objective variation of the wavelength can be registered when a source is moving through a matter. Let us take a look at the following figure (1): 


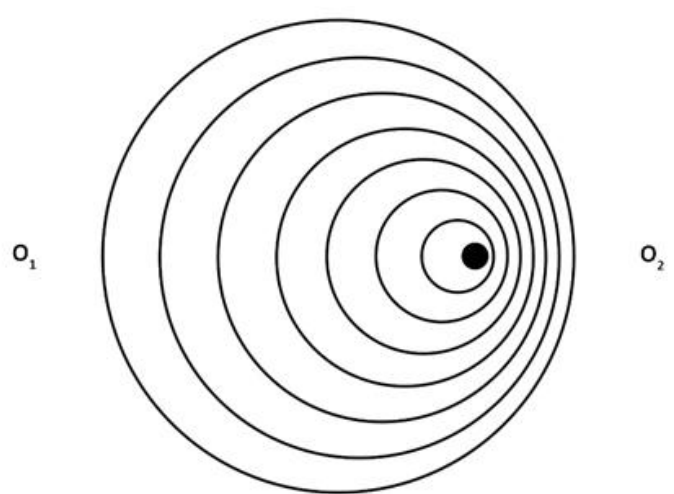

Fig. 1: Velocity of Source Relative to Observers is to the Right.

As we can see, the movements of the source trough the matter produce a real objective increasing or decreasing of the wavelength, so that the observer receives an increased or decreased frequency. Besides, we note that the waves are not "emitted" from the source, but are disturbs of the matter in which the source is moving through. Which means that the waves are made of the matter in which they propagate and not of that of the moving source? In case of decrease of wavelength, the following formula is used:

$f=f_{0} \frac{v}{v-v_{s, r}}$

f0 = originally emitted; $\mathrm{f}=$ observed; $\mathrm{v}=$ velocity of waves through matter; $\mathrm{vs}, \mathrm{r}=$ velocity of source with regard to observers.

Now, we examine the case when the observer is moving to a stationary source of waves (fig. 2):

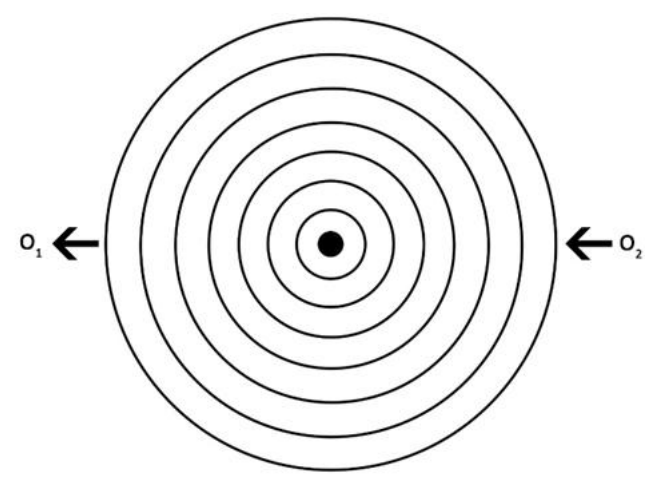

Fig. 2: Observers Move To and From Source

By observing the here above figure is evident that we are observing a completely different phenomenon than that described in fig. 1 . What we can see in fig. 2 is that there is no increasing or decreasing of wavelength, but a difference in frequency the observer subjectively records due to the relative motion between the latter and the source. In this case the frequency observed is simply to calculate by this formula:

$\mathrm{f}^{\prime} 0=\frac{(\mathrm{Vu}-\mathrm{V}) \mathrm{f}}{\mathrm{Vu}}$

( $\mathrm{f}^{\prime} \mathrm{o}=$ frequency obs; $\mathrm{Vu}=$ speed of the waves trought matter; $\mathrm{V}=$ relative speed between source and observer)

Which obviously brings to a different result whit regard to (1): we have to consider that we are dealing with two different phenomena from each other, so that we also expect two different results. In the first case, we are dealing with a real objective increasing or decreasing of wavelength. In the second case we can observe an increasing or decreasing of frequency subjectively perceived by an observer due to the relative motion.

Dealing with the Doppler-Fiseau effect, relatively to electromagnetic waves, we have to remind that there is no difference in results when the source is moving to a stationary observer (1), objectively producing a different wavelength and when are the observers moving to a stationary source (2) producing its original wavelength, but a subjectively perceived, different frequency by the observer. The result connecting the two different cases, dealing with Doppler-Fiseau schift, is produced by the formula 2 and not by 1 .

Which means: in this case we have to input the differences in observed frequency to the increased or decreased relative motion between source and observer: $(\mathrm{C}-\mathrm{V})$.

On base of this specification about the Doppler Effect, we hint the following statement:

The redshift is not consequence of moving away of energy sources from each other's, but of a slowdown of speed of light coming to us from big distances. Which means: the farther the sources are from us, the slower the lights reach us. 


\section{Results}

Starting from this premise, we have to establish there is no difference between the wavelengths emitted the wavelength observed:

$\left(\lambda_{\text {emit }}=\lambda_{\text {obsv }}=\lambda\right)$

The measure that in this case has been perceived as a decreasing of wavelength is in fact that of a decreasing of the frequency the observer records due to the global slowing down of the wavy fluxes, with regard to the observer self.

We take as an example the quasar known as 3C-273:

The hydrogen Ballmer-alpha line of this body has been calculated in a wavelength of $\left(\lambda_{\text {emit }}\right) 760 \mathrm{~nm}$.

The wavelength observed $\left(\lambda_{\text {obsv }}\right)$ in $656 \mathrm{~nm}$.

From the angle of a slowdown of light, we have to consider the observed wave-length as a observed decreased frequency:

$\left(\lambda_{\text {obs }}=\mathrm{f}_{\text {obs }}\right)$

The calculation of the redshift $(\mathrm{z})$ based on this difference will be:

$\lambda=760 \mathrm{~nm}$

$\mathrm{f}_{\text {obs }}=656$

$\mathrm{z}=\frac{\text { fobs }-\lambda}{\lambda}=0,1336$

Starting from the principle of constancy of light-speed and consequent decreasing of wavelength due to the Dopplershift, the calculation of the redshift of this body based on frequency has been calculated in $\mathrm{z}=0,1585$ and needs a relativity correction to reach the observed result.

Differently, when we calculate the value on base of a slowdown effect (v), and consequently that of the observed decreased light speed $\left(\mathrm{C}_{\text {obsv }}\right)$ :

$\mathrm{v}=(\mathrm{cz})=(300.000)(0,1336)=41.400 \mathrm{Km} / \mathrm{s}$

$\mathrm{C}_{\mathrm{obs}}=(\mathrm{c}-\mathrm{v})=300.000-41.400=258.600 \mathrm{~km} / \mathrm{s}$

And:

$\mathrm{f}_{\text {obsv }}=\frac{(\mathrm{C}-\mathrm{V}) \lambda}{\mathrm{c}}=656$

Which is the real obtained result.

\section{Conclusion}

In conformity to the premise and the results which sustain its consistency, will be possible to hint that the universe is not expanding. The relation distance/redshift would give us the image of a globally static universe and as logical consequence, the fact that light coming from the most distant celestial bodies undergoes a slowdown which is directly proportional to the distance from us: the bigger the distance that light fluxes have to travel to reach us, the stronger the slowdown, the sharper the redshift.

The statement made by Hubble about the principle of universal expansion makes the ground of the "Big-Bang Theory". When this ground would be missing, there will be any other reason to sustain the universe sourced by an original explosion of an enormous concentration of matter. This will exonerate us from the embarrassment to find or imagine explanations with regard to where and how each celestial body finds the necessary energy to move from all others in a constant acceleration. In some cases their speed of moving away could proximally reach that of light.

To remain on field of Thought Physics, when we want to compare electromagnetic waves to the classic ones, or rather, to the propagations of disturbs of any kind of matter produced by a foreign item, (like explicated by fig.1)we should imagine that electromagnetic waves are rippling of the transporting magnetic fields. It is well known that magnetic fields are sensible to gravity. It would be a question to research if the sensibility that magnetic fields undergo by gravity can only have influence on the bogging of the fields or also may be responsible for a progressive slowdown of light fluxes travelling through big distances. Experiments carried out on ground of "slow-light" [as solved by 1;2; 3; 4] have proven the concrete possibility to operate a slowdown of light fluxes.

A concrete and consistent alternative to open a new field of research about the origin and dynamics of our universe.

\section{References}

[1] Mark Zentile Department of Physics, Durham University, South Road, Durham, DH1 3LE, UK (Dated: June 28, 2012. Available on: http://massey.dur.ac.uk/maz/MarkZentileReportEV.pdf

[2] Lene Hau "Physics Central" 1999. Available on: http://www.physicscentral.com/explore/people/hau.cfm

[3] Ryan M. Camacho, Michael V. Pack, and John C. Howell // Physical Review A 13 June 2006. Available on: http://pra.aps.org/abstract/PRA/v73/i6/e063812

[4] Mehmet Fatih Yanik and Shanhui Fan Ginzton Laboratory, Stanford University, Stanford, California 94305/ Phys. Rev. Lett. Date Sept. 10, 2003. Available on: http://arxiv.org/ftp/quant-ph/papers/0312/0312027.pdf 